\title{
Haptic identification of common objects: Effects of constraining the manual exploration process
}

\author{
SUSAN J. LEDERMAN \\ Queen's University, Kingston, Ontario, Canada \\ and \\ ROBERTA L. KLATZKY \\ Carnegie Mellon University, Pittsburgh, Pennsylvania
}

\begin{abstract}
In this article, we address the effects on haptic recognition of common objects when manual exploration is constrained by using two kinds of rigid links - sheaths (Experiment 1A) and probes (Experiments $1 \mathrm{~B}$ and 2). The collective effects of five different constraints are considered, including three from previous research (i.e., reducing the number of end effectors, wearing a compliant finger cover, and splinting the fingers; Klatzky, Loomis, Lederman, Wake, \& Fujita, 1993) and from two current constraints (i.e., wearing a rigid finger sheath and using a rigid probe). The resulting impairments are interpreted in terms of the loss of somatosensory information from cutaneous and/or kinesthetic inputs. In addition, we relate the results to the design of haptic interfaces for teleoperation and virtual environments, which share some of the same reduction of sensory cues that we have produced experimentally.
\end{abstract}

In the present article, we will consider how well people can haptically identify common objects when manual exploration is constrained. The constraints imposed in this study were produced by imposing a rigid link between the skin and the object, in the form of a sheath over the finger or a probe held in the hand. Any constraint that forms an intermediate barrier between skin and objects can be described as producing remote (or indirect) perception. Such intermediaries serve to constrain haptic exploration by reducing the cutaneous and/or kinesthetic inputs available. In earlier work (Klatzky, Loomis, Lederman, Wake, \& Fujita, 1993), manual exploration has been constrained in several other ways that did not involve using a rigid link. In this article, we integrate the results of the earlier Klatzky et al. (1993) study with those of the present study to further our understanding of haptic object identification by direct and remote touch. In addition, we relate the result to the design of haptic interfaces for teleoperation and virtual environments, which share some of the same cue reductions that we have produced experimentally. Our article arises from two separate but related themes in previous research on haptic perception.

This research was supported by grants from NSERC (Natural Sciences \& Engineering Council of Canada) and IRIS (Canadian Federal Institute for Robotics and Intelligent Systems). Some of the results of Experiment 1B were published in Lederman and Klatzky (1998). Correspondence concerning this article should be addressed to S. Lederman, Queen's University, Department of Psychology, Kingston, ON, K7L 3N6 Canada (e-mail: lederman@psyc.queensu.ca).

\section{Excellence of Haptic Recognition of Common Objects}

The first theme involves the fact that people are excellent at haptically recognizing common objects and that material, as well as shape, cues are critical to this endeavor. Some years ago Klatzky, Lederman, and Metzger (1985) showed that people are remarkably proficient at identifying common objects with the use of their bare hands. Blindfolded subjects manually identified 100 common objects with almost $100 \%$ accuracy, typically within only 2-3 sec per object. Clearly, people are quite capable of identifying objects by touch alone. Moreover, there are numerous situations in everyday life when we are forced to do so. For example, we frequently reach into our pockets or bags stuffed with visually occluded objects to successfully remove a set of keys, a wallet, or a lipstick. In darkened environments we commonly reach for known objects, such as an alarm clock or a cup.

In a recent chapter, we (Klatzky \& Lederman, 2000) addressed the nature of processing during haptic object recognition in relation to visual processing of objects. It is now widely accepted that the earliest stages of visual object recognition involve the extraction of spatial information in the form of oriented edges, which are combined to produce low-level object primitives or features. Biederman (1987), for example, proposed a set of volumetric primitives known as geons. He suggested that common objects are represented in terms of the spatial synthesis of several geons, with relative size and position maintained. Recognition involves comparing the object in question with representations of different object categories stored in mem- 
ory. Is it possible that the same general processes of edge extraction and spatial synthesis of primitives apply to the haptic recognition of objects?

In its most direct application, such an approach proves difficult inasmuch as the haptic system processes the orientation of edges in space rather ineffectively. Lederman and Klatzky (1997) showed that although subjects were able to detect the presence or absence of an edge within a haptic display after only a few milliseconds of contact, it took them considerably longer to spatially process edges in terms of orientation. The results from performing haptic search tasks involving different object properties suggest that the haptic system can evaluate edges relatively quickly and accurately if processed intensively (i.e., in terms of magnitude); in contrast, performance is considerably poorer when edges are processed spatially (i.e., in terms of a spatial reference system). Not surprisingly, then, the haptic system is compromised - slow and prone to error - when processing edges contained in fingertip-sized 2-D patterns (see, e.g., Lederman \& Klatzky, 1997; Loomis, 1990) and in larger raised 2-D spatial displays (Lederman, Klatzky, Chataway, \& Summers, 1990; Magee \& Kennedy, 1980).

Haptic recognition of fully 3-D objects exclusively on the basis of edge information is relatively poor as well. To evaluate the use of spatial information for 3-D haptic object recognition, Klatzky et al. (1993) created conditions in which subjects were restricted to using edge information by fixing rigid objects to a supporting surface (no weight or part-motion cues) and by requiring subjects to wear a compliant glove that further deprived them of texture and thermal cues. Accuracy ranged from $74 \%$ to $93 \%$ for gloved single-finger and gloved whole-hand manual exploration, respectively; the corresponding response times ranged from $45 \mathrm{sec}$ down to $16 \mathrm{sec}$, respectively.

Why do people show poor performance in haptic apprehension of edge information? We propose two reasons. First, processing of local edges with the fingertip, as compared with the eye, is limited by the fingertip's relatively coarse spatial resolving capacity. Second, the manner in which people explore 2-D patterns and 3-D objects further constrains haptic spatial processing. Lederman and Klatzky (1987) have shown that people typically extract edges by using variants of two hand-movement patterns, or exploratory procedures (EPs). When precise spatial details about edges are desirable, people must perform a contour-following EP, which involves tracing along edges. This forced sequential exploration slows observers considerably by many seconds and thus imposes a heavy memory load on temporal integration that frequently leads to error. Subjects are confined to contour following when exploring raised 2-D patterns. When examining fully 3-D objects, an enclosure EP (or grasp) can also be performed. This procedure is considerably faster $(\sim 2 \mathrm{sec})$ than the contour-following EP in that it provides simultaneous information about edges; however, the sparse local information provided by use of the fingers and palm is much less spatially precise.
Up to this point, we have addressed the deficiencies with which the haptic system processes spatial edge information and the geometric properties derived from such low-level information. In contrast, the haptic system is highly efficient - both fast and accurate - when processing object properties on the basis of the materials from which objects are constructed (e.g., texture, compliance, or thermal properties). An object's material properties are usually independent of the object's geometric features, with the exception of weight, which is a hybrid property determined by both density and volume. Lederman and Klatzky (1987) showed that when subjects freely derive information about each of the categories pertaining to a designated material property, a specific EP is optimal for a given property, in that it provides the most precise information about that property. Each of the EPs used to obtain precise material, as opposed to geometric, information can be performed very quickly (i.e., $\sim 2 \mathrm{sec}$ ). People have also proved capable of making coarse binary discriminations with respect to material properties (i.e., rough/ smooth, hard/soft, and warm/cool) when limited to a very brief haptic glance - an initial contact lasting only about $200 \mathrm{msec}$ (Klatzky and Lederman, 1995). In contrast, subjects took considerably longer to differentiate geometric properties related to relative position, relative orientation of a 2-D bar, and relative slant of a 3-D surface (Lederman \& Klatzky, 1997).

Now let us return to the highly accurate recognition performance typically observed when people explore common multiattribute objects with their bare hands (Klatzky et al., 1985). There may be at least two ways in which such good performance is achieved despite subjects' relatively poor ability to extract spatial edge information (Klatzky \& Lederman, 2000) and construct geometric descriptions of objects.

First, shape may not be the only source of diagnostic information pertaining to the successful classification of common objects. Classification can be at several different levels, as was noted by Rosch (1978). The wooden object that people use to sit on can be classified functionally as furniture (superordinate level); however, it can also be classified as a chair (basic level), or even more exclusively as a desk chair (subordinate level). Typically, people classify objects at the basic level, which mainly differentiates categories in terms of their shape. Lederman and Klatzky (1990) required subjects to select properties, in order of importance, from a closed list that they would use to identify the objects at either basic or subordinate levels, as designated in each trial. The full set of stimulus object names was selected so that at the subordinate level, a range of object properties would be considered critical. Note that it was indeed possible to generate subordinatecategory names for which material properties - not just shape - were judged to be most critical (e.g., stale bread). Moreover, material properties, particularly texture, often proved diagnostic of categories at the basic, as well as subordinate, level. The findings support the possibility that 
people might use material properties to successfully classify some common objects.

Second, even if shape is most diagnostic of an object's category, recognition performance may well be enhanced if people use material properties as an auxiliary source of information. In Experiment 1 of the Klatzky et al. (1993) study, subjects' hands were covered in thick gloves to limit the contribution of material cues and to force haptic object recognition based on object geometry. In Experiment 2, the fingertips of the gloves were cut away, allowing subjects to touch the objects directly and to extract additional information about differences in texture, compliance, and thermal conductivity. As was predicted, recognition accuracy and response times both improved in Experiment 2. Subsequently, Klatzky and Lederman (1995) showed that when contact time was restricted to about $200 \mathrm{msec}$, subjects were able to identify common objects more successfully when the most diagnostic attribute was texture than when it was shape. Collectively, these studies confirm that material properties can serve as a valuable secondary source of information about objects that can compensate for poor haptic recognition via geometric properties such as shape.

\section{Constraining Manual Exploration Impairs Haptic Perception}

The second theme motivating this research is that haptic perception is impaired when manual exploration is constrained. Constraints arise frequently in everyday activities. People are forced to explore objects and their properties in ways that are less than optimally effective, such as, for example, when wearing some form of skin cover like a glove or when using a hand that has sustained peripheral nerve damage or that is excessively cold. In each case, the available cutaneous and/or kinesthetic information is reduced relative to when manual exploration is totally unconstrained.

Klatzky et al. (1993) examined the effects of three different constraints on manual exploration. Each impaired the haptic recognition of common objects relative to an unconstrained-hand control condition. Table 1 shows the nature of the constraints used in that study and the associated loss(es) that would be expected in somatosensory information, cutaneous and/or kinesthetic. As is shown in the table, the specific constraints reduced sensory inputs in different ways: by reducing the number of fingers used, by rigidly splinting the fingers, and by wearing a compli- ant glove. The effects of these constraints were to reduce cutaneous information in the form of spatial, temporal, or thermal cues, and/or to reduce kinesthetic information. (We do not differentiate between spatial and temporal kinesthetic cues in the table, because any constraint on hand movement is likely to have both spatial and temporal effects.) Note that manual exploration can be simultaneously constrained in more than one way, resulting in multiple sources of somatosensory loss.

With respect to the manipulations in the Klatzky et al. (1993) study, reducing the number of fingers used from five to one reduced the subject's haptic spatial field of view. Splinting the finger(s) prevented subjects from molding their finger(s) to the object contours and thereby reduced the spatial and temporal kinesthetic information about object geometry normally provided by finger articulation during a grasp (enclosure EP). Requiring subjects to wear a compliant glove that covered the entire hand (including fingertips) reduced or eliminated spatially distributed force patterns and thermal cues to material. Haptic object recognition became poorer when the haptic field of view was reduced and when subjects were prevented from performing an enclosure by splinting (Experiment 1). Restoring normal fingertip function to the fingertips (by cutting away the fingertips of the glove) improved haptic object recognition, presumably by making available information about the objects' material properties (Experiment 2).

In the present study, we required subjects to manually explore common objects by using a rigid link in the form of a finger sheath or a hand-held probe. This study expands our previous work in two ways. First, it extends our investigation of haptic object recognition under conditions of constrained exploration by adding a new form of constraint — rigid links. Second, it extends our work on the effects of rigid linkages on haptic perception, in which object recognition has not heretofore been considered as a task domain. Rigid links serve to constrain manual exploration by reducing cutaneous spatial deformation and thermal cues, and kinesthetic cues. Our work has suggested, however, that rigid links should leave vibratory information relatively intact. We found that a rigid finger sheath had no effect on vibratory thresholds (Lederman \& Klatzky, 1999), and people were only moderately impaired in judging surface texture via rigid links (see, e.g., for fingertip sheath, Lederman \& Klatzky, 1999; for probe, Klatzky \& Lederman, 1999; Klatzky, Lederman,

Table 1

Constraints on Manual Exploration and Somatosensory Cues With Significant Reduction

\begin{tabular}{|c|c|c|c|c|c|}
\hline \multirow{2}{*}{$\begin{array}{c}\text { Constraint } \\
\text { Number }\end{array}$} & \multirow{2}{*}{$\begin{array}{c}\text { Nature } \\
\text { of Manipulation }\end{array}$} & \multicolumn{3}{|c|}{ Cutaneous } & \multirow{2}{*}{$\begin{array}{c}\text { Kinesthetic Spatia } \\
\text { and Temporal }\end{array}$} \\
\hline & & Spatial & Temporal & Thermal & \\
\hline 1 & Reduced number of end effectors & $x$ & & & $x$ \\
\hline 2 & Compliant covering & $x$ & $x$ & $x$ & \\
\hline 3 & Rigid finger splinting & & & & $\times$ \\
\hline 4 & Rigid finger sheath & $\times$ & & $\times$ & $\times$ \\
\hline 5 & Rigid probe & $\times$ & & $\times$ & $\times$ \\
\hline
\end{tabular}


Hamilton, Grindley, \& Swendsen, 2003; Lederman \& Klatzky, 1999).

\section{The Present Study}

We used a single end effector in all experimental conditions - specifically, a bare finger partially enclosed in a rigid finger sheath, the hand holding a rigid probe, or a single bare finger. The use of a single end effector, as opposed to multiple fingers, constitutes Constraint 1 in Table 1 . The use of a sheath or probe constitutes Constraints 4 and 5 , respectively.

How well can people haptically recognize common objects when constrained by the use of these rigid links, as compared with the three other constraints listed in Table 1? Experiment 1 evaluated the effects of two types of rigid links on haptic object recognition. In Experiment 1A, subjects were constrained to the use of the distal portion of the finger encased in a rigid sheath (Constraints 1 and 4); the control condition was a single bare finger (Constraint 1 ). In Experiment 1B, subjects used two rigid probes with different tip sizes (Constraints 1 and 5); performance obtained with each was compared with that obtained with the other, and with that of a single bare finger (Constraint 1). In Experiment 2 , we used a constrained haptic identification task and selected common objects that explicitly varied in terms of whether their most diagnostic feature was texture or shape (Lederman \& Klatzky, 1990). Subjects explored the objects by using a rigid probe with a small tip that retained vibrations (Constraints 1 and 5) or a single bare finger (Constraint 1 ). We predicted that the cue reduction effected by the rigid probe and finger sheath would impair recognition of objects more than the bare finger would, particularly when shape, as opposed to texture, was the most diagnostic feature. At the end of this article, we will consider the implications of the studies on constraining manual exploration for the design of haptic interfaces for teleoperation and virtual environment systems. The research was approved by the local ethics committee.

\section{EXPERIMENTS 1A AND 1B Haptic Object Identification via Rigid Links Versus a Bare Finger}

In Experiment 1A, the subject's index finger was covered with a rigid plastic sheath surrounding the distal portion of the finger to eliminate or reduce cutaneous, thermal, and kinesthetic cues. A glove with the single finger portion cut away allowed us to compare performance via this rigid link with that of the bare finger. Experiment $1 \mathrm{~B}$ employed the rigid probes used in the initial experiment in our research on remote perception of surface texture (Klatzky \& Lederman, 1999). In Experiment 1B, subjects were asked to haptically identify common objects either remotely by using the small- or large-tipped probe or directly with the bare finger. Both the sheath (Experiment 1A) and the probes (Experiment 1B) leave vibratory cues relatively unaffected but eliminate the spatial, temporal, and thermal cues available from cutaneous contact. Both types of rigid links reduce kinesthetic cues. The sheath provides input from object-related finger movement above the distalinterphalangeal (DIP) joint, whereas the probe must be grasped. Thus, kinesthetic cues related to the object's shape arise only from joints proximal to the fingers. Not surprisingly, we expected, given these cue reductions, that people would be impaired at object recognition with the rigid links relative to the bare finger. The additional question was how much impairment would result, and indeed, whether it would be possible to achieve accurate recognition with these links.

\section{Method}

\section{Experiment 1A}

Subjects. A total of 16 subjects received course credit for participating. None had any known sensorimotor deficits in their hands.

Stimuli and Apparatus. The stimuli consisted of 38 common objects listed in the Appendix (e.g., book, pliers) that were used by Klatzky et al. (1993). Each object was mounted on the center of a $30.5 \times 145.7 \mathrm{~cm}$ board, which was placed in front of the subject in a prototypical orientation. Two were used for practice.

Sheaths. For each subject, a plastic sheath (Friendly Plastic) shaped like a tube was molded to the surface of the index finger from warm plastic. The process took approximately $5 \mathrm{~min}$. The cooled rigid sheath fit tightly around the finger from the tip to the middle of the proximo-interphalangeal joint (PIP), thus preventing articulation at the DIP. On the basis of five sample measurements, the mean thickness was approximately $1.5 \mathrm{~mm}$.

Procedure. After the mold was created, the subject was blindfolded. He or she put on a fabric knit glove with the index finger cut away; the glove served merely to prevent accidental contact with the object by the skin of the hand. For the sheath condition, the rigid sheath was placed over the subject's index finger. During the practice that preceded the start of the formal experiment, the experimenter presented two objects from the stimulus set; the subjects used the end effector designated for the first condition (bare or sheathed finger).

On each trial, the subject's hand was guided to the object, at which point he or she could begin exploring. The subject was instructed to stop whenever he or she knew the object and to give its name. If the object could not be identified within $2 \mathrm{~min}$, the experimenter terminated the trial and recorded a 2 -min response time. No multiple choice options were provided, because we wished to study free naming. The subject was instructed not to tap the object deliberately to determine its sound. He or she wore earphones that played a tape recording of the sounds of a rigid implement tapping the objects, to mask any inadvertent sound cues. Sound-attenuating covers were placed over the earphones. The subject was instructed to feel the object only with the index finger and to keep the rest of the fingers curled into the palm. The glove masked any incidental contact.

Experimental design. A within-subjects design was used. The objects were randomly divided into four groups, which were always presented in the same order; the order of objects within a group was randomized for each subject. All possible sequences of the two finger conditions and the two sheath conditions were used across subjects as equally as possible given the number of subjects, meaning that both conditions were repeated twice.

\section{Experiment 1B}

Subjects. A total of 20 students received course credit for participating. The subjects had no known sensorimotor deficits in their hands.

Stimuli and Apparatus. The same set of common objects as that in Experiment 1A was presented.

Probes. The two Delrin probes have been described in detail in Klatzky and Lederman (1999). Delrin is a material that is somewhat 
slippery, with high-impact strength and abrasion resistance over a fairly wide temperature range. The cylindrical shaft lengths were $15 \mathrm{~cm}$. The large probe terminated in a half-ellipsoid shape that was slightly rounded at the tip. The small probe ended in a conical shape that was slightly rounded at the tip. The measured contact diameters for the small and large probes were approximately 2 and $4 \mathrm{~mm}$, respectively.

Procedure. The subject was first blindfolded and fitted with a fabric knit glove with the top $2.0 \mathrm{~cm}$ of each fingertip removed. The remaining portion of the glove covered $4.5 \mathrm{~cm}$ of the proximal portion of the thumb, $5.0 \mathrm{~cm}$ of the index finger, and $5.5,5.0$, and $3.0 \mathrm{~cm}$ of the remaining fingers, respectively. The subject was unconstrained with respect to how the probe was held; however, he or she was instructed to grasp it away from the tip and to use only the tip to effect contact. Most of the subjects held the probe as they would a pencil. The subject's hand was guided so that the probe tip contacted the object, at which point the subject could explore the object. In all other respects, the procedure was the same as that used in Experiment 1A.

Experimental design. A within-subjects design was used. The objects were randomly divided into four groups. The groups were always presented in the same order but were assigned to different probe conditions across subjects; the order in which objects within a group were presented was randomized for each subject. Each subject identified two groups of stimuli with the small probe and another two groups with the large probe. All possible orders of the four probe conditions were used across subjects as equally as possible given the number of subjects available.

\section{Results}

Experiment 1A. Mean accuracy was $91.7 \%(S D=$ $7.9 \%)$ for the bare finger and $41.7 \%(S D=14.8 \%)$ for the sheathed finger. The corresponding mean response times were $31.3 \mathrm{sec}(S D=13.4)$ and $83.1 \mathrm{sec}(S D=18.2)$, respectively. With subjects as the unit of observation, $t$ tests showed that both differences were highly significant $[t(15)=13.67$ and 19.12 for accuracy and response time, respectively, both $p \mathrm{~s}<.0001]$. The corresponding tests with object as the unit of observation also indicated statistical significance $[t(15)=10.19$ and 14.54 for accuracy and response time, respectively, both $p \mathrm{~s}<.0001]$. The performance values for Experiment 1A are summa- rized in Table 2, together with results from previous relevant studies and the results from Experiment 1B.

Experiment 1B. Mean accuracy was $41.4 \%(S D=$ $12.0 \%)$ and $38.9 \%(S D=17.2 \%)$ for the small- and largetipped probes, respectively; the corresponding mean response times were $85.6 \mathrm{sec}(S D=9.9)$ and $85.2 \mathrm{sec}(S D=$ 16.9). These values are considerably lower than are the levels obtained in Experiment 1A and by Klatzky et al. (1993) for real objects with the bare index finger (Klatzky et al., 1993, Experiment 2: mean accuracy, 84\%; mean response time, $22.8 \mathrm{sec}$ ) and with the gloved index finger (Klatzky et al., 1993, Experiment 1: mean accuracy, 74\%; mean response time, $45.2 \mathrm{sec}$ ). The probe recognition levels are very close to the value achieved with the sheath in Experiment 1A. According to $t$ tests, the differences between the two probes did not approach significance. The results are summarized in Table 2.

Integration with Klatzky et al. (1993). To assess the effects of different constraints on a single end effector, we conducted a factor analysis that included, by stimulus object, Klatzky et al.'s (1993) mean accuracy and response time data from the single-finger conditions with real objects along with the new mean accuracy and response time data corresponding to the bare-finger and sheathed-finger conditions in the present Experiment $1 \mathrm{~A}$ and the smalland large-probe conditions in the present Experiment 1B. The purpose of conducting this analysis was to determine whether common constraints would lead conditions from different experiments to load on a common factor. The rotated factor loadings are shown in Table 3 for each of the 12 variables. For purposes of clarity, cells with values $<.25$ are not shown.

In a three-factor solution, it appeared that conditions with common constraints were pooled, as was expected. It accounted for a total of $81.8 \%$ of the variance. Factor 1 appears to specifically reflect variation in the accuracy and response times when the rigid links (probes and sheaths) were used. The fact that the sheath (Experiment 1A) and

Table 2

Summary of Experimental Results on Haptic Recognition of Common Objects From Previous Studies and the Present Experiment 1

\begin{tabular}{|c|c|c|c|c|c|c|}
\hline \multirow[b]{2}{*}{ Study } & \multirow[b]{2}{*}{ Variable } & \multicolumn{3}{|c|}{ Constraints* } & \multirow{2}{*}{$\begin{array}{c}\text { Accuracy } \\
(\%)\end{array}$} & \multirow{2}{*}{$\begin{array}{l}\text { Response } \\
\text { Time (sec) }\end{array}$} \\
\hline & & 12 & 3 & 45 & & \\
\hline Klatzky et al. (1985) & whole hand & \multicolumn{3}{|c|}{ unconstrained } & $96-99$ & $2-3 * *$ \\
\hline \multirow{7}{*}{ Klatzky et al. (1993) } & whole hand, no glove & \multicolumn{3}{|c|}{ unconstrained } & 95 & 6 \\
\hline & whole hand, gloved, fingertips removed & \multicolumn{3}{|c|}{ unconstrained } & 93 & 10 \\
\hline & whole hand, gloved & \multirow{2}{*}{\multicolumn{3}{|c|}{2}} & 93 & 16 \\
\hline & five fingers splinted & & & & 90 & 18 \\
\hline & five fingers splinted + gloved & \multicolumn{3}{|c|}{23} & 90 & 25 \\
\hline & one finger splinted & \multicolumn{3}{|l|}{1} & 85 & 23 \\
\hline & one finger splinted + gloved & 12 & 3 & & 74 & 45 \\
\hline \multirow[t]{2}{*}{ Present Experiment 1A } & one finger & \multicolumn{3}{|l|}{1} & 92 & 31 \\
\hline & one finger in rigid sheath & 1 & & 4 & 42 & 83 \\
\hline \multirow[t]{2}{*}{ Present Experiment 1B } & probe (small) & \multicolumn{3}{|r|}{5} & 41 & 86 \\
\hline & probe (large) & \multicolumn{3}{|l|}{1} & 39 & 85 \\
\hline
\end{tabular}

Note-*Constraint numbers correspond to those shown in Table 1. **The response time mode is reported in this study. In all other studies, the response time mean is reported. 
Table 3

Rotated Factor Analysis Solution

\begin{tabular}{|c|c|c|c|c|c|}
\hline Study & Variable & Measure & Factor 1 & Factor 2 & Factor 3 \\
\hline Klatzky et al. (1993, Experiment 1) & gloved finger & accuracy & & & .915 \\
\hline Klatzky et al. (1993, Experiment 1) & gloved finger & response time & .494 & & -.768 \\
\hline Klatzky et al. (1993, Experiment 2) & bare finger & accuracy & & .844 & \\
\hline Klatzky et al. (1993, Experiment 2) & bare finger & response time & .320 & -.654 & \\
\hline Present Experiment 1A & bare finger & accuracy & & .856 & \\
\hline Present Experiment 1A & bare finger & response time & .380 & -.556 & \\
\hline Present Experiment $1 \mathrm{~A}$ & sheathed finger & accuracy & -.575 & .252 & .451 \\
\hline Present Experiment $1 \mathrm{~A}$ & sheathed finger & response time & .819 & & -.279 \\
\hline Present Experiment 1B & small probe & accuracy & -.854 & .249 & \\
\hline Present Experiment 1B & small probe & response time & .928 & & \\
\hline Present Experiment 1B & large probe & accuracy & -.867 & & \\
\hline Present Experiment 1B & large probe & response time & .902 & & -.264 \\
\hline Percentage of variance explained* & & & 42.5 & 21.7 & 17.6 \\
\hline Total percentage of variance explained* & & & 81.8 & & \\
\hline
\end{tabular}

Note_- ${ }^{*}$ Calculation includes all loadings $>.25$.

two probes (Experiment 1B) produced very similar accuracy and response times suggests that the sheath may function in the same way as a large probe. These conditions left vibratory cues intact but eliminated spatial cutaneous and thermal cues. Factor 2 differentially reflects the use of the bare finger in the Klatzky et al. (1993, Experiment 2) study and in the present Experiment 1A. Finally, Factor 3 appears to account for variation in responses when subjects explored common objects with a single finger that was covered in a compliant glove (Klatzky et al., 1993, Experiment 1). This condition reduced not only spatial cutaneous and thermal cues, as do the probes and sheath, but also vibratory cues. In short, the three factors correspond to relatively different levels of cue availability with respect to the single finger: (1) cutaneous temporal cues only, (2) full cutaneous and kinesthetic cues, and (3) kinesthesis only. Note that Klatzky et al. (1993) found that single-finger conditions were related to distinct factors from multiple-finger conditions, as would be expected in that the single finger limits both cutaneous spatial and kinesthetic cues (see Table 1). It fails to provide the spatially distributed cutaneous pattern across the palm and the kinesthetic cues caused by the molding of multiple fingers to the contours of an object that are provided by fullhand exploration.

\section{EXPERIMENT 2 \\ Identifying Common Objects at Basic and Subordinate Levels by Using a Bare Finger, a Finger With Compliant Cover, or a Rigid Probe}

The subjects in Experiment 2 performed the constrained, common-object identification task that had been used by Lederman and Klatzky (1990). From that study, we selected objects whose most diagnostic attribute was texture versus shape or size (shape/size). We focused on those properties because we expected that performance clearly would be differentially affected by the nature of the constraint on manual exploration.

Manual exploration was constrained by having the subjects explore by using the rigid probe with the small spher- ical tip from Experiment 1B (Constraints 1 and 5) or, alternatively, by exploring with the bare finger (Constraint 1 ). The small probe served to eliminate all normal cutaneous spatial patterns and thermal cues, leaving or perhaps amplifying the temporal patterns (vibration) in the skin; kinesthetic inputs from finger articulation would no longer be available either. On the basis of the constraints imposed by the probe, we predicted not only that recognition would be generally poorer with the probe than with the bare-finger condition but also that, more specifically, probe recognition should be particularly impaired for shape/sizediagnostic objects. Because the textural properties were still transmitted through the probe by vibration, performance should suffer less for texture-diagnostic objects. Thus, as compared with the difference between the probe and the bare finger for shape/size-diagnostic items, the difference with respect to texture-diagnostic items should be small. We did not predict that people should perform equally well with the probe and finger for texture-diagnostic items, since subjects might encounter particular physical difficulty in using a very small, rigid tip to sequentially trace along the narrow object contours. This would impair performance with the probe.

\section{Method}

Subjects. The subjects were 32 students who participated for course credit. They were drawn from the same population as in Experiment 1 . They had no known sensorimotor deficits in their hands and described themselves as right-handed.

Stimuli. The stimuli consisted of 36 common objects. There were 4 practice items and 32 experimental items (see the Appendix). Within each of these sets, texture was the most diagnostic attribute (MDA) for half of the objects, and shape or size was the MDA for the other half. Of the practice items, two occurred in positive trials (in which the named object was the object presented) and two in negative trials (in which the named object was not the one presented). Of the experimental items within each MDA, 10 were positive and 6 negative. Each subject responded to the practice items followed by the experimental items, presented in random order.

Procedure. The subjects were assigned in alternating order to one of two groups, which differed in the type of end effector used. One group explored with the bare index finger. A second group explored with the rigid probe with the small tip from Experiment 1B. 
The subject was blindfolded and wore sound-attenuating earphones through which played a tape of the probe contacting the objects to mask any inadvertent sound cues. Each subject wore a cottonknit glove that masked incidental contact with an object. For the bare-finger condition, the end of the index finger of the glove was cut away, exposing the finger above the PIP joint. For the probe condition, the thumb, index, and middle fingers of the glove were cut away completely, allowing the skin to be exposed over the entire area contacting the probe. The subject was specifically instructed to hold the probe in the same way as a pencil, so that its shaft contacted the index finger just below the metacarpo-phalangeal joint. The subjects were instructed to hold the fingers not used for exploration against the palm and not to tap on the object to hear how it sounded.

At the beginning of each trial, the experimenter asked a question of the form, "Is this [basic-level name] a [subordinate-level name]?" The subject was instructed to indicate whether he or she did not know the object that had been named at the subordinate level; if so, the trial was aborted. No additional cues were given. If the subject knew the object category, the experimenter guided his or her end effector to the object (to an edge or upper surface with no distinguishing features) and started the timer. The observers were instructed to answer the question as soon as they could, and they were told that if they could not answer within $1 \mathrm{~min}$, the trial would be terminated. The experimenter recorded correct/incorrect responses $(1 / 0)$ and corresponding response times (in seconds).

\section{Results}

$\boldsymbol{d}^{\prime}$ analyses. A two-factor mixed-design ANOVA was performed using $d$ 's, which were calculated for each subject on the basis of the responses to the positive and negative questions. In the analyses, subject was the unit of observation. A small adjustment permitted us to include subjects with $0 \%$ (scored as .005) or 100\% (scored as .995) error levels in the statistical analysis. The betweensubjects factor was end effector, with two levels (bare finger or probe). The within-subjects factor was diagnostic property, with two levels (texture and shape/size). Because the adjustment artificially inflates the values of actual mean $d^{\prime}$ s in the ANOVA, in Table 4 we report instead the $d^{\prime}$ values calculated on the mean proportions for the positive (hits) and negative (false alarms) questions. The effect of end effector was highly significant $[F(1,30)=$ 48.25, $p<.0001]: d^{\prime}$ was higher for the bare finger than for the rigid probe. Neither the effect of diagnostic property nor the two-way interaction was statistically significant. Both the accuracy $\left(d^{\prime}\right)$ and response time data (considered next) for Experiment 2 are reported in Table 4.

Response times. A mixed-factor three-way ANOVA was performed on the response time data. The betweengroups factor was end effector with two levels (bare finger or probe); the two within-subjects factors were diag-

Table 4

Summary of the Performance Results From the Present Experiment 2

\begin{tabular}{lcccccccc}
\hline & & \multicolumn{3}{c}{ Accuracy $\left(d^{\prime}\right)$} & \multicolumn{5}{c}{ Response Time $(\mathrm{sec})$} \\
\cline { 3 - 4 } \cline { 7 - 9 } Variable & Constraints* & $\mathrm{T}$ & $\mathrm{S}$ & & $\mathrm{T}+$ & $\mathrm{T}-$ & $\mathrm{S}+$ & $\mathrm{S}-$ \\
\hline One finger & 1 & 2.64 & 2.36 & 6.6 & 5.2 & 6.8 & 6.0 \\
Probe (small) & 1,5 & 1.10 & 1.32 & & 15.4 & 15.2 & 22.0 & 15.8 \\
\hline
\end{tabular}

Note- ${ }^{*}$ Constraint numbers correspond to those shown in Table 1. T, texture; $\mathrm{S}$, shape; $\mathrm{T}+$, texture positive; $\mathrm{T}-$, texture negative; $\mathrm{S}+$, shape positive; $\mathrm{S}-$, shape negative. nostic property with two levels (texture or shape) and question type, also with two levels (positive or negative). The three-way interaction indicated a highly elevated response time for one cell, the positive probe trials in which shape/size was diagnostic $[F(1,30)=9.06, p<.007]$, which led to the main effects [end effector, $F(1,30)=42.36$, $p<.0001$; diagnostic property, $F(1,30)=25.58, p<$ .0001 ; question, $F(1,30)=9.06, p<.007]$ and two-way interactions [diagnostic property $\times$ end effector, $F(1,30)=$ $11.83, p<.002$; question $\times$ end effector, $F(1,30)=6.08$, $p<.05$; question $\times$ diagnostic property, $F(1,1)=7.20$, $p<.05$ ] all being statistically significant.

\section{Discussion}

The results obtained in Experiment 2 lend additional support to the distinction between direct and remote object recognition. The $d^{\prime}$ and response times for remote recognition of texture and shape/size-diagnostic objects via the small probe were consistently lower than those obtained directly with the bare finger. In this case, with respect to the single bare finger, thermal and spatially distributed force patterns and spatial and temporal kinesthetic cues were eliminated or substantially reduced. We further predicted that, relative to the bare finger, the probe would specifically impair shape/size-based more than texturebased common object recognition, owing to the loss of local spatially distributed force patterns on the skin.

The prediction was not confirmed for the $d^{\prime}$ data. In hindsight, we recognize that with few exceptions, the texturediagnostic questions related to differences in material, as opposed to texture alone. The use of the probe, which eliminated thermal as well as spatial deformation cues, would likely have been more detrimental to material-based, as opposed to shape/size-based, object recognition than had been anticipated. Performance with the probe was equally impaired relative to the bare finger for shape/size-based versus texture-based questions.

However, we observed a marked discrepancy in response times with the probe. More specifically, response times for shape/size positive questions were substantially longer than those for shape/size negative questions, which were the same as those for both texture positive and texture negative questions. This result indicates that in order to achieve equivalent accuracy levels with the probe for texture and shape/size questions, people had to explore longer for shape/size. The difficulty with extracting shape and size particularly affected the time to answer positive questions. Presumably this is because the negative items could be disconfirmed by a single feature, whereas the positive items had to be explored more exhaustively. For texture-based questions, the probe generated diagnostic vibratory information. Because the diagnostic texture features in this experiment were homogeneously distributed across the queried area of the object (i.e., the shape features), a difference in response times between texture positive and texture negative questions would not be expected, nor did one occur. And since texture homogeneity commonly occurs within the fingertip contact area for most common objects, 
we believe this finding to apply more generally. The finding that the response times for negative shape/size questions were close to those for the texture questions further confirms that the shape/size disconfirmations were found by means of local exploration.

\section{GENERAL DISCUSSION}

People manually perceive and manipulate the concrete world both directly with their bare hands and remotely via intervening links between the skin and the external surface. When haptic exploration is entirely unconstrained, a wealth of cutaneous and kinesthetic inputs is available for further processing. Normally spatial, temporal, and thermal information from skin contact can be used together with kinesthetic information from static positioning and movement of the fingers, hand, and arm. In the present study, we have examined the consequences for haptic object recognition of limiting manual exploration to the use of rigid intermediate links (i.e., sheaths and probes). When exploration is constrained in this way, cutaneous cues consisting of spatial deformation patterns and thermal cues are reduced if not eliminated, leaving only static and transient changes in pressure. Kinesthetic inputs are also reduced because of limited or no finger articulation.

In the present study, direct haptic exploration involved the use of a single, bare finger (Experiments 1A, 1B, and 2). In remote haptic exploration, a rigid fingertip sheath (Experiment $1 \mathrm{~A}$ ) and rigid probes with differently sized tips (Experiments 1B and 2) were used. On the basis of the collective results from previous studies as well as the present one, we will now consider the effects of constraining manual exploration in five different ways on the haptic recognition of common objects by direct and remote touch. These effects are summarized in Tables 1-4.

\section{Direct Haptic Exploration}

Whole hand (unconstrained). Earlier comparative data have clearly indicated the efficacy of using the whole bare hand during unconstrained exploration of objects that varied primarily in their geometric features (Klatzky et al., 1985; Klatzky et al., 1993, baseline data). This condition made available all spatially, temporally, and thermally distributed cutaneous cues from multiple end effectors as well as kinesthetic inputs that provide additional valuable information about 2-D and 3-D object geometry. With whole-hand exploration, people could execute a grasp by using the highly stereotypical hand movement pattern (EP) known as enclosure. This EP provides a relatively quick means of obtaining the coarse structural information required for identifying common objects, particularly at the basic level (Lederman \& Klatzky, 1987).

Reduced number of end effectors (Constraint 1 ). When subjects were constrained by exploring the same objects with a single, bare finger (Klatzky et al., 1993; present Experiment 1A), performance declines. The subjects were prevented from taking advantage of the full range of 3-D information normally available when the fingers mold to object contours. Although recognition accuracy declined only a little, the response times increased substantially. Presumably, reduced performance was the result of the subjects' being forced to sequentially examine the common objects identifiable primarily by their 3$\mathrm{D}$ geometric features.

Rigid splinting (Constraint 3). In addition, constraining exploration by attaching a rigid splint to a single, bare finger (Klatzky et al., 1993) probably constrained exploration about the same as did using the bare, unsplinted finger, inasmuch as the subjects did not bend the finger in either case. Because only a single finger was permitted under both constraints, the subject was forced to extract contour information by exploring the object's structure sequentially, with no opportunity to mold to its contours. The associated exploratory procedure, contour following, is sequential and slow (Lederman \& Klatzky, 1987). Hence, it was more difficult to temporally integrate sequentially extracted inputs into an overall geometric representation of the object. A similar difficulty arises when blind individuals haptically explore tangible graphics displays (e.g., pictures, maps, graphs, etc.) that present spatial information in the form of raised 2-D lines.

\section{Remote Haptic Exploration}

Compliant finger cover (Constraint 2). Covering the index finger with a compliant layer caused the least information reduction among the remote end effector conditions that we have investigated in the present as well as past studies. Gloving the fingertip(s) in the Klatzky et al. (1993, Experiment 1) study was originally intended to reduce cutaneous cues to surface texture, thus restricting most available information to global object contours via sequential exploration. The compliant glove reduced the accuracy of haptic object recognition when people explored with one finger but not when they used five fingers. It also increased the response times for both one- and five-finger conditions; not surprisingly, the one-finger impairment proved considerably greater.

Rigid finger sheath (Constraint 4). In the present study, relative to accuracy for a bare-finger control, covering a single finger with a rigid sheath (Experiment $1 \mathrm{~A}$ ) reduced recognition accuracy by a factor of 2.2 at the same time as it increased the mean response time by a factor of 2.7. The rigid sheath retained vibratory and net contact force cues while eliminating thermal and spatially distributed force cues that were correlated with surface geometry. The rigid sheath would also have reduced kinesthetic inputs at the DIP joint, although the subjects tended to keep their index fingers outstretched. Lederman and Klatzky (1999) have previously shown that eliminating spatially distributed force patterns on the skin with the same rigid sheaths substantially impaired performance in several other tasks, including spatial resolution (two-point touch threshold), pressure sensitivity, perceived orientation of a raised bar, and the size threshold for detecting a 


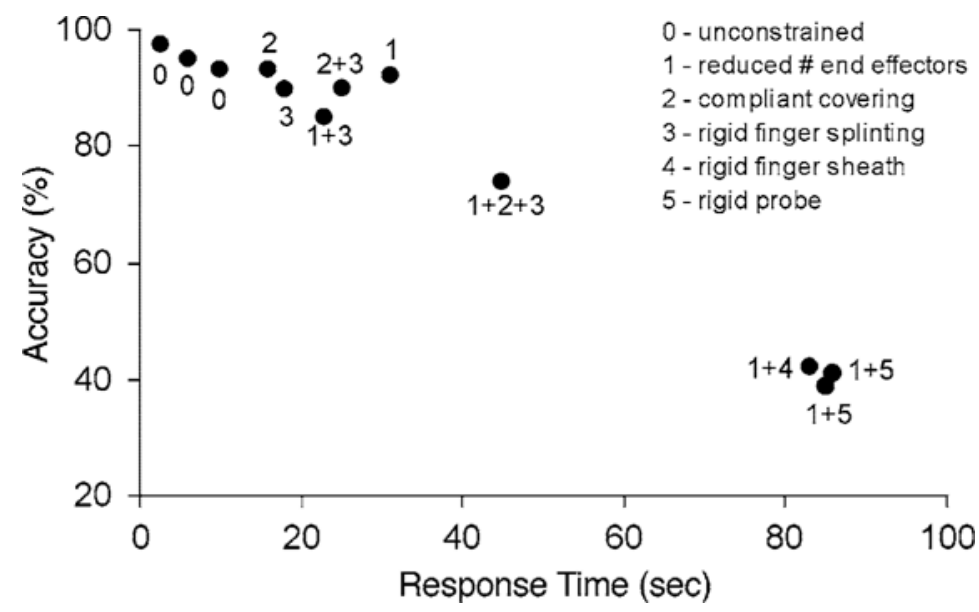

Figure 1. Recognition accuracy (\%) as a function of response time (in seconds) for various constraints on manual exploration, presented singly and/or in some combination. The constraints are listed both by name and by number in the legend. The data are drawn from the experimental conditions in Klatzky, Loomis, Lederman, Wake, and Fujita (1993) and in Experiments 1A and 1B of the present study. The "+" sign indicates the existence of multiple simultaneous constraints.

simulated lump in simulated tissue via palpation. Note that texture discrimination was only moderately impaired, as is explained by the fact that the rigid sheath left vibrotactile thresholds unchanged.

Rigid probes (Constraint 5). Manual exploration was even further limited when the subjects remotely explored the set of common objects used by Klatzky et al. (1993) with the use of rigid probes with small or large spherical tips (Experiment 1B). As with the rigid sheaths (Experiment $1 \mathrm{~A}$ ), relative to the accuracy of the appropriate barefinger control, accuracy dropped by a factor of $\sim 2.2$ (Experiment $1 \mathrm{~B}$, both probes). However, response times increased considerably with the rigid probes than with the sheath - that is, by a factor of 4 (Experiment 1B, both probes) relative to the response times in the bare-finger condition. Presumably, such constraints limit access to thermal cues and to precise textural and geometric details, particularly the latter. The reduction of kinesthetic inputs was greater for the probe than for the sheath used in Experiment $1 \mathrm{~A}$ inasmuch as the former eliminated all normal finger articulation.

Figure 1 graphically summarizes the collective effects of constraining manual exploration on object recognition performance. Accuracy (\%) and the corresponding response times (in seconds) are shown for the five constraints, singly and/or in some combination, based on the experimental conditions used in the Klatzky et al. (1993) study and in Experiments $1 \mathrm{~A}$ and $1 \mathrm{~B}$ of the present study. To summarize, the unconstrained conditions (0) clearly show the highest accuracies and shortest response times. Rigidly splinting a single finger (3) and a compliant finger cover (2) both similarly impede performance least, by slightly reducing accuracy and by increasing response time. Reducing the number of end effectors from the whole hand to a single finger (1) also slightly reduces accuracy but increases response time considerably more than does either the compliant cover (2) or the rigid finger splint (3). Combining the compliant cover (2) and rigid splint (3) constraints further increases response time, whereas combining the reduced number of end effectors (1) and rigid splint (3) constraints decreases accuracy. Combining all three constraints $(1,2$, and 3$)$ markedly reduces both accuracy and response time still further. Finally, combining either the rigid sheath (4) or the rigid probe (5) constraints with the reduced number of end effectors (1) constraint has strikingly negative consequences for recognition performance in terms of accuracy and response time.

The factor analysis (Table 3 ) that was performed on the results from the earlier Klatzky et al. (1993) study as well as the present one (Experiments 1A and 1B) allows us to contrast performance in a number of conditions that similarly restricted manual exploration to the use of a single end effector (Constraint 1). Factor 1 highlights Constraint 4 (Experiment 1A, rigid sheath) and Constraint 5 (Experiment $1 \mathrm{~B}$, rigid probe), both of which involve remote perception via rigid intermediate links. Factor 3 highlights remote perception via compliant intermediate links (Klatzky et al., 1993, Experiment 1). As the rigidity of the intermediate links decreases, recognition accuracy increases and response time decreases (Table 2). Compliant surfaces reduce spatially distributed force patterns and dampen vibrations on the skin, which may result in impaired extraction of texture features. Rigid linkages eliminate the spatial force patterns while leaving, if not amplifying, vibrotactile cues. Rigid links also prevent/limit finger articulation, further reducing kinesthetic cues to object 
geometry. Factor 2 highlights direct perception via the bare finger. Performance was best in this case, presumably because of the advantage of full cutaneous and kinesthetic cues within the finger.

\section{Implications for Remote Interactions With Real and Virtual Environments}

Recently, the haptic research community has devoted considerable attention to the haptic perception and dexterous manipulation of remote environments. Remote interactions with real environments require the design and use of haptic interfaces, mechanical systems that sense forces in remote environments and deliver those forces to the hand of the user in the form of a haptic display accessed via a rigid link. Haptic interfaces are now being designed for use in a number of teleoperation and virtual environment application domains, such as virtual medical training systems, space repairs, and CAD applications that require the user to perceive and identify objects and their properties (e.g., geometric or material). The characteristics of the real or virtual inputs will therefore vary considerably across application domains, depending on what objects/features are being represented and on the available modes of manual exploration. The present results may be used to plan interface design in a more principled manner by making design choices on the basis of the general consequences of constraining manual exploration in one or more ways (see Table 1 and Figure 1).

Most current haptic interfaces involve single, point contact interactions with the environment. In the case of the commercially available PHANTOM, subjects grasp a pencil-like probe or insert the forefinger into a thimble and move the end effector around within the workspace of the interface. The position of the tip of the end effector is sensed, and forces are delivered through the tip to simulate contact with virtual objects that vary in material and geometric properties. We have argued that a simple rigid probe can serve as an experimental model for the more complex systems that involve point contact achieved by moving the stylus or finger inserted in a thimble. Such interfaces severely constrain haptic exploration by limiting the normally rich cutaneous information to highly localized vibrations and the kinesthetic information to kinematic and dynamic cues produced by moving the rigid link. With respect to Table 1 , such point contact haptic interfaces therefore limit manual exploration by means of Constraint 5 (probe) or Constraint 4 (thimble) plus Constraint 1 (reduced number of end effectors).

The present article has highlighted the perceptual costs of constraining haptic exploration to a single point. The user can likely detect the presence of edges and judge relative compliance and vibration-based textures. However, the loss of spatially distributed cutaneous inputs may impair performance of spatial tasks that require the tracking of contours and the processing of very fine spatial patterns. Moreover, sequential point contact exploration imposes a heavy memory load on object processing that will likely limit the effective temporal integration of inputs related to 3-D structure (see, e.g., Kirkpatrick \& Douglas, 2002). Even two-point contact distributed across two hands is not suitable for extracting metric properties or for generalizing across changes in body orientation (Klatzky, Lederman, \& Langseth, 2003).

Multiple contact points, at least one per finger, would seem critical for effecting a quick multi-fingered grasp to provide simultaneous, albeit spatially coarse, kinesthetic information about 3-D object structure. Only a sensorized glove would provide whole-hand contact that includes the fingers and palm. Although palm contact is desirable, we believe the additional consequences of adding it would be relatively minor and limited to interactions with large objects. However, the use of whole-hand exoskeleton haptic interfaces that are mounted on the dorsal surface of the hand may constrain manual exploration to the extent that they confine finger movement. The present study has highlighted the fact that Constraint 3, on its own, impairs object-recognition performance primarily by increasing response time, although not as extensively as does using a single bare finger.

\section{REFERENCES}

Biederman, I. (1987). Recognition-by-components: A theory of human image understanding. Psychological Review, 94, 115-147.

KirkPatricK, A. E., \& Douglas, S. A. (2002). Application-based evaluation of haptic interfaces. Proceedings of the IEEE 10th Symposium of Haptic Interfaces for Virtual Environments and Teleoperator Systems (pp. 32-39).

KlatZKy, R. L., \& Lederman, S. J. (1995). Identifying objects from a haptic glance. Perception \& Psychophysics, 57, 1111-1123.

Klatzky, R. L., \& Lederman, S. J. (1999). Tactile roughness perception with a rigid link interposed between skin and surface. Perception \& Psychophysics, 61, 591-607.

KLATZKY, R. L., \& LEDERMAN, S. J. (2000). L'identification haptique des objets significats [The haptic identification of common objects]. In Y. Hatwell \& E. Gentaz (Eds.), Toucher pour connaitre [Touching in order to know] (pp. 109-128). Paris: Presses Universitaires de France.

Klatzky, R. L., Lederman, S. J., Hamilton, C., Grindley, M., \& SWENDSEN, R. H. (2003). Feeling textures through a probe: Effects of probe and surface geometry and exploratory factors. Perception \& Psychophysics, 65, 613-631.

KLATZKY, R. L., LEDERMAN, S. J., \& LANGSETH, S. (2003). Watching a cursor distorts haptically guided reproduction of mouse movement. Journal of Experimental Psychology: Applied, 9, 224-235.

Klatzky, R. L., Lederman, S. J., \& Metzger, V. A. (1985). Identifying objects by touch: An "expert system." Perception \& Psychophysics, 37, 299-302.

KLATZKY, R. L., LoOmis, J. M., Lederman, S. J., Wake, H., \& Fujita, N. (1993). Haptic identification of objects and their depictions. Perception \& Psychophysics, 54, 170-178.

LEDERMAN, S. J., \& KLATZKY, R. L. (1987). Hand movements: A window into haptic object recognition. Cognitive Psychology, 19, 342-368.

Lederman, S. J., \& KlatzKy, R. L. (1990). Haptic classification of common objects: Knowledge-driven exploration. Cognitive Psychology, 22, 421-459.

LEDERMAN, S. J., \& KLATZKY, R. L. (1997). Relative availability of surface and object properties during early haptic processing. Journal of Experimental Psychology: Human Perception \& Performance, 23, 1680-1707.

LeDerman, S. J., \& KlatzKy, R. L. (1998). Feeling through a probe. Proceedings of the ASME International Mechanical Engineering Congress: Dynamic Systems and Control Division, 64, 127-131.

Lederman, S. J., \& KlatZKy, R. L. (1999). Sensing and displaying spa- 
tially distributed fingertip forces in haptic interfaces for teleoperator and virtual environment systems. Presence: Teleoperators \& Virtual Environments, 8, 86-103.

Lederman, S. J., KlatzKy, R. L., Chataway, C., \& Summers, C. D. (1990). Visual mediation and the haptic recognition of two-dimensional pictures of common objects. Perception \& Psychophysics, 47, 54-64.

Loomis, J. M. (1990). A model of character recognition and legibility.
Journal of Experimental Psychology: Human Perception \& Performance, 16, 106-120.

MAGEE, L., \& KENNEDY, J. (1980). Exploring pictures tactually. Nature, 283, 287-288.

Rosch, E. (1978). Principles of categorization. In E. Rosch \& B. Lloyd (Eds.), Cognition and categorization (pp. 27-48). Hillsdale, NJ: Erlbaum.

\section{APPENDIX}

List of Objects

\begin{tabular}{|c|c|c|c|c|c|}
\hline Trial Type & MDA & Question Type & Basic Level & Subordinate Level & Actual Item Presented \\
\hline \multirow[t]{4}{*}{ Practice } & & positive & cereal & cheerios & \\
\hline & & & pencil sharpener & plastic pencil sharpener & \\
\hline & & negative & button & shirt button & large button \\
\hline & & & paper clip (metal) & plastic paper clip & metal paper clip \\
\hline \multirow[t]{32}{*}{ Experimental } & texture & positive & bowl & wooden bowl & \\
\hline & & & glasses frame & plastic glasses frame & \\
\hline & & & pie plate & aluminum foil pie plate & \\
\hline & & & dish towel & terrycloth dish towel & \\
\hline & & & sandpaper & coarse sandpaper & \\
\hline & & & flower pot & clay flower pot & \\
\hline & & & clothespin & plastic clothespin & \\
\hline & & & tape & scotch tape & \\
\hline & & & shirt & cotton shirt & \\
\hline & & & disposable cup & disposable styrofoam cup & \\
\hline & & negative & doorknob & wooden doorknob & glass doorknob \\
\hline & & & paintbrush & wooden-handled paintbrush & plastic paintbrush \\
\hline & & & corduroy & wide corduroy & narrow corduroy \\
\hline & & & tape & masking tape & electrical tape \\
\hline & & & shirt & silk shirt & flannel shirt \\
\hline & & & disposable cup & disposable cup & plastic disposable cup \\
\hline & shape/size & positive & hook & picture hook & \\
\hline & & & fork & salad fork & \\
\hline & & & key & filing cabinet key & \\
\hline & & & sandals & child's sandal & \\
\hline & & & can & sardine can & \\
\hline & & & watch & woman's watch & \\
\hline & & & padlock & keyed padlock & \\
\hline & & & glass & brandy glass & \\
\hline & & & noodle & elbow macaroni noodle & \\
\hline & & & milk carton & half-pint milk carton & \\
\hline & & negative & soap & hotel soap & bathroom soap \\
\hline & & & battery & nine-volt battery & flashlight battery \\
\hline & & & cup & measuring cup & coffee mug \\
\hline & & & glass & wine glass & drinking glass \\
\hline & & & noodle & spiral noodle & shell noodle \\
\hline & & & milk carton & pint milk carton & quart milk carton \\
\hline
\end{tabular}

Note-MDA, most diagnostic attribute; question type positive, the named object was the object presented; question type negative, the named object was not the one presented.

(Manuscript received June 9, 2003;

revision accepted for publication September 1, 2003.) 